\title{
Concentration of antibodies against Porphyromonas gingivalis is increased before the onset of symptoms of rheumatoid arthritis
}

Linda Johansson ${ }^{1 \dagger}$, Natalia Sherina ${ }^{2 \dagger}$, Nastya Kharlamova², Barbara Potempa ${ }^{3}$, Barbro Larsson ${ }^{2}$, Lena Israelsson², Jan Potempa ${ }^{3,4}$, Solbritt Rantapää-Dahlqvist ${ }^{1^{*+}}$ and Karin Lundberg ${ }^{2 \dagger}$

\begin{abstract}
Background: The periodontal pathogen Porphyromonas gingivalis is hypothesized to be important in rheumatoid arthritis (RA) aetiology by inducing production of anti-citrullinated protein antibodies (ACPA). We have shown that ACPA precede RA onset by years, and that anti-P. gingivalis antibody levels are elevated in RA patients. The aim of this study was to investigate whether anti-P. gingivalis antibodies pre-date symptom onset and ACPA production.

Methods: A case-control study (251 cases, 198 controls) was performed within the Biobank of Northern Sweden. Cases had donated blood samples ( $n=422$ ) before the onset of RA symptoms by 5.2 (6.2) years (median (interquartile range)). Blood was also collected from 192 RA patients following diagnosis. Antibodies against $P$. gingivalis virulence factor arginine gingipain B (RgpB), and a citrullinated peptide (CPP3) derived from the $P$. gingivalis peptidylarginine deiminase enzyme, were analysed by ELISA.

Results: Anti-RgpB IgG levels were significantly increased in pre-symptomatic individuals (mean \pm SEM; $152.7 \pm$ 14.8 AU/ml) and in RA patients $(114.4 \pm 16.9 \mathrm{AU} / \mathrm{ml})$, compared with controls $(p<0.001)$. Anti-CPP3 antibodies were detected in $5 \%$ of pre-symptomatic individuals and in $8 \%$ of RA patients, with elevated levels in both subsets $(4.33 \pm 0.59$ and $9.29 \pm 1.81 \mathrm{AU} / \mathrm{ml}$, respectively) compared with controls $(p<0.001)$. Anti-CPP3 antibodies followed the ACPA response, with increasing concentrations over time, whilst anti-RgpB antibodies were elevated and stable in the pre-symptomatic individuals with a trend towards lower levels after RA diagnosis.

Conclusions: Anti-P. gingivalis antibody concentrations were significantly increased in RA patients compared with controls, and were detectable years before onset of symptoms of RA, supporting an aetiological role for $P$. gingivalis in the development of RA.

Keywords: Porphyromonas gingivalis, Antibodies, Arginine gingipainB, Citrullinated peptide, Rheumatoid arthritis, Predating onset, Anti-citrullinated protein/peptide antibodies

Abbreviations: ACPA, Anti-citrullinated protein/peptide antibodies; anti-CCP, Antibodies against citrullinated peptide antibodies; CCP1, filaggrin307-324, citrullinated at position 13; anti-CEP-1, antibodies against a-enolase5-21 citrullinated at positions 9 and 15; anti-cFib $\beta 36-52$, antibodies against fibrinogen $\beta 36-52$ citrullinated at position 44; AU, Arbitrary units; CCP, cyclic citrullinated peptide; Cl, Confidence interval; CPP3, Cyclic citrullinated peptide 3; ELISA, Enzyme-linked (Continued on next page)
\end{abstract}

\footnotetext{
* Correspondence: solbritt.rantapaa.dahlqvist@umu.se

${ }^{\dagger}$ Equal contributors

'Public Health and Clinical Medicine/Rheumatology, Umeå University, Umeå,

Sweden

Full list of author information is available at the end of the article
} 
(Continued from previous page)

immunosorbent assay; HLA, Human leukocyte antigen; IQR, Interquartile range; OR, Odds ratio; PAD, Peptidylarginine deiminase enzyme; PD, Periodontitis; PTPN22, Protein tyrosine phosphatase non-receptor type 22; RA, Rheumatoid arthritis; RF, Rheumatoid factor; RgpB, Arginine gingipainB; ROC, Receiver operating characteristic; RPP3, Argininecontaining control peptide; SD, Standard deviation; SE, Shared epitope; SEM, Standard error of mean

\section{Background}

Rheumatoid arthritis (RA), a complex chronic inflammatory disease with a worldwide prevalence of $0.5-1.0 \%$ [1], is characterized by production of anti-citrullinated protein/peptide antibodies (ACPA) in the majority of patients and persistent inflammation in the synovial tissue of the joints leading to destruction of cartilage and bone [2-4]. The aetiology of RA remains unknown, although a complex interplay exists between genetic and environmental factors [5, 6]. Chronic periodontitis (PD), which is the world's commonest inflammatory disease often resulting in destruction of alveolar bone and tooth loss, has been suggested as an environmental determinant for the occurrence and severity of RA [7-9].

Elucidation of the potential aetiological link between PD and RA has progressed $[8,10,11]$ and a number of studies have identified similarities between these two diseases that possibly explain the epidemiological association. Both PD and RA display systemic markers of inflammation (e.g. C-reactive protein and pro-inflammatory cytokines) $[6,12,13]$, and an association with $H L A-D R B 1$ alleles [14] and smoking [15, 16] has been described for both RA and PD. Furthermore, citrullinated proteins have been detected in both rheumatoid joints and inflamed gingival tissue, as well as in other tissues in relation to inflammatory conditions [17-19]. However, an association between PD and established RA could not be confirmed in one of our recent publications [20].

Moreover, data from one of our other studies suggest that the oral pathogen Porphyromonas gingivalis, rather than PD, may be linked to the development of RA [21]. $P$. gingivalis is a common periodontal pathogen associated with PD $[22,23]$, and is the only prokaryote known to express an endogenous peptidylarginine deiminase enzyme (P.PAD), a virulence factor capable of citrullinating human and bacterial proteins, including autocitrullination [24-26]. P.PAD interacts with another major virulence factor, arginine gingipainB (RgpB), an arginine-specific extra-cellular protease expressed on the surface of the bacterial outer membrane [24, 25]. RgpB is essential for $P$. gingivalis to citrullinate peptides; that is, only after degradation by RgpB can P.PAD convert peptidylarginine into peptidylcitrulline $[24,26]$.

It is hypothesized that citrullination by $P$. gingivalis causes a chronic exposure of citrullinated peptides in the inflamed periodontium, possibly leading to a break of immune tolerance in genetically susceptible individuals and subsequent production of ACPA. Epitope spreading, induced by molecular mimicry and cross-reactivity with citrullinated epitopes exposed in the joint, could lead to progression of chronic RA [27-29]. ACPA appear many years before the onset of RA [30,31], suggesting that the initial immune dysregulation occurs long before symptoms of RA develop, outside the joints, potentially at mucosal sites such as the gingival tissue [17, 27, 32]. Citrullinated P.PAD has been demonstrated to be a target of the ACPA response [25] and we recently demonstrated that elevated anti-RgpB antibody levels have a stronger association with RA than smoking [21], identifying $P$. gingivalis as a potential mechanistic link between PD and RA. Importantly, in the same study we could also show that anti-RgpB IgG levels were significantly increased in sera from PD patients compared with periodontally healthy individuals, supporting antiRgpB IgG as a surrogate marker for oral infection by P. gingivalis.

This study investigated whether raised anti- $P$. gingivalis antibody levels precede onset of symptoms of RA and the ACPA response in order to elucidate the role of $P$. gingivalis as a potential trigger of autoimmunity and the development of RA. Importantly, because we have no data on periodontal status in our cohorts, we have only focused on P. gingivalis, not on PD, in the present study. Consequently, we analysed the antibody response to RgpB and CPP3, a citrullinated peptide derived from P.PAD, in blood samples collected prior to the onset of symptoms and at the diagnosis of RA. Identifying a potential mechanism capable of breaking immunological tolerance at the earliest stage of disease would provide an insight into the aetiopathology of RA, and could indicate new clinical therapies and interventions.

\section{Methods}

\section{Study population}

A case-control study was performed within the Medical Biobank of Northern Sweden and the Maternity cohort. The cohorts within the Medical Biobank are populationbased health surveys, and all habitants of Västerbotten county are continuously invited to participate. Information concerning recruitment, blood sampling and storage conditions has been described previously [30]. The Maternity cohort is based on blood samples collected from pregnant women being screened for immunity to rubella [30]. To identify individuals having donated 
blood samples prior to onset of symptoms of RA, the register of patients fulfilling the 1987 classification criteria for RA [33] was linked to those of the Medical Biobank and the Maternity cohort.

The study included 251 pre-symptomatic individuals (58 men/193 women, mean age \pm SD $50.5 \pm 11.9$ years) who had donated a total of 422 plasma/serum samples (375 from the Biobank cohorts and 47 from the Maternity cohort) at different time points pre-dating onset of RA symptoms. From the same cohorts, 198 (31 men/167 women, mean age \pm SD $49.3 \pm 14.8$ years) populationbased controls (173 from the Biobank cohorts and 25 from the Maternity cohort), matched for sex and age, with sufficient plasma/serum volumes, were identified. The median time pre-dating the onset of RA symptoms was 5.2 years (interquartile range 6.2). At least one sample was identified for each of the 251 pre-symptomatic individuals; two samples were identified for 92 individuals $(36.6 \%)$, three samples for 46 individuals (18.3\%), four samples for 22 individuals ( $8.8 \%$ ), five samples for nine individuals (3.6\%) and six samples for two individuals $(0.8 \%)$. At the time of RA diagnosis ( $\leq 12$ months of symptoms), 192 patients (144 females/48 males) donated blood samples at the early arthritis clinic. One hundred and fifty-three of these early RA patients were identified within the group of pre-symptomatic individuals because they had donated blood samples before onset of symptoms (median 5.6 years, IQR 6.3). Data detailing periodontal status or treatment were not available for these cases. The participants gave their written informed consent and the Regional Ethics Committee at Umeå University approved the study.

Smoking status was defined as ever smoker (including former and current smokers), current smoker or never smoker. Because information on being either former or current smoker was lacking for a number of cases, we present data for both groups. Of the pre-symptomatic individuals, $64 \%(160 / 250)$ were ever smokers and $25.6 \%(55 / 215)$ were current smokers, while $49.2 \%$ (89/ 181) of the controls were ever smokers and $13.1 \%$ (18/ 137) were current smokers. HLA-DRB1 shared epitope (SE) alleles (0101/0401/0404/0405/0408) and PTPN22
1858C/T polymorphism were genotyped as described previously [34, 35]. Demographic data for the three groups are presented in Table 1.

\section{Antibody analysis}

Using in-house ELISAs as described previously [21, 25], all plasma/serum samples were analysed blinded for antibodies against the RgpB protein purified from $P$. gingivalis [36], a synthetic cyclic citrullinated peptide (CPP3) derived from P.PAD and the corresponding arginine-containing control peptide (RPP3) (Innovagen AB, Lund, Sweden). Serial dilutions of antibody-positive serum pools (antiRgpB and anti-CPP3 IgG, respectively) were included on all ELISA plates to generate standard curves in order to compare antibody concentrations between cases analysed on different ELISA plates. All antibody levels are presented as arbitrary units $/ \mathrm{ml}(\mathrm{AU} / \mathrm{ml})$.

Treating anti-CPP3 IgG as a "classical" ACPA, a cut-off value for antibody positivity was defined using receiver operating characteristic (ROC) curves, based on the antiCPP3 IgG responses in RA patients and controls. The cutoff value for positivity was set at $>29.19 \mathrm{AU} / \mathrm{ml}$, giving a specificity of $96 \%$. No cut-off value could be assigned for the anti-RgpB IgG response, due to a lack of data regarding PD status of the study subjects.

The plasma/serum samples included in this study had been analysed previously for the presence of antibodies against cyclic citrullinated peptides (CCPs), using the anti-CCP2 ELISA assay (Euro Diagnostica, Malmö, Sweden), and different ACPA fine specificities (e.g. cfibrinogen $\beta 36-52$ (Fib $336-52$, citrullinated at position 44), $\alpha$-enolase5-21 (CEP-1, citrullinated at position 9 and 15) and cfilaggrin307-324 (CCP1, citrullinated at position 13)) using a microarray based on the ImmunoCAP ISAC system (PhaDia AB, Uppsala, Sweden) [37]. Analysis of rheumatoid factor (RF) of IgM isotype was performed using the EliA assay on the Phadia 2500system according to the manufacturer's instructions (Phadia GmbH, Freiburg, Germany). The cut-off value with the optimum sensitivity and specificity was that with $96 \%$ specificity.

Table 1 Descriptive data of the pre-symptomatic individuals, patients with RA and controls

\begin{tabular}{llll}
\hline & Pre-symptomatic individuals ${ }^{\mathrm{a}}(n=251)$ & RA patients $(n=192)$ & Controls $(n=198)$ \\
\hline Female sex, $n(\%)$ & $193(76.9)$ & $144(75.0)$ & $167(84.3)$ \\
Age, mean \pm SD years & $50.5(11.9)^{\mathrm{b}}$ & $56.5(11.3)$ & $49.3(14.8)$ \\
Ever smoker, $n(\%)$ & $160 / 250(64.0)$ & $129(67.2)$ & $89 / 181(49.2)$ \\
HLA-DRB1 SE positive $^{c}, n(\%)$ & $166 / 250(66.4)$ & $122 / 192(63.5)$ & $61 / 171(35.7)$ \\
PTPN22 1858 T carrier, $n(\%)$ & $86 / 250(34.4)$ & $63(32.8)$ & $32 / 173(18.5)$ \\
\hline
\end{tabular}

Pre-symptomatic individuals were defined as individuals before the onset of symptom of RA

${ }^{\mathrm{b}}$ Mean age, as calculated for all samples when collected, pre-symptomatic individuals: $n=422$

CHLA-DRB1 shared epitope (SE) alleles were defined as 0101/0401/0404/0405/0408

$R A$ rheumatoid arthritis, $S D$ standard deviation 


\section{Statistical analysis}

Continuous data were compared using a non-parametric Mann-Whitney U-test/Wilcoxon signed rank test including two groups, and a Kruskal-Wallis test including several groups. The chi-square test or Fisher's exact test was used when analysing categorical data. Correlation analysis was performed using Spearman's rank correlation. Because of the lack of a cut-off value for the antiRgpB antibody response, we stratified the anti-RgpB antibody concentrations into above or below the 75th percentile in the analyses. Logistic regression analysis was used to identify associations between antibodies and risk factors in the development of RA adjusted for age and sex. Associations were presented as odds ratios (ORs) with $95 \%$ confidence interval (CI). Standard methods were used for analysing interactions [38]. All adjustments were based on previously performed studies and hypothesis. The statistical analyses were performed using SPSS 23.0 software (Chicago, IL, USA).

\section{Results}

The anti-RgpB antibody response and anti-CPP3 antibody response in pre-symptomatic individuals, RA patients and controls

The concentration of anti-RgpB IgG was significantly increased in RA patients (mean \pm SEM 114.4 $\pm 16.9 \mathrm{AU} /$ $\mathrm{ml}$ ) and in particular in pre-symptomatic individuals, calculated using all 422 samples $(152.7 \pm 14.8 \mathrm{AU} / \mathrm{ml})$ or one sample per individual (when more than one sample was available, the sample closest to symptom onset was chosen) (133.4 $\pm 16.2 \mathrm{AU} / \mathrm{ml}$; data not shown), compared with control subjects $(82.2 \pm 12.1 \mathrm{AU} / \mathrm{ml}, p<0.001$ for all three groups) (Fig. 1a).

The anti-CPP3 IgG levels were significantly increased in RA patients (mean \pm SEM 9.29 $\pm 1.81 \mathrm{AU} / \mathrm{ml}$ ) compared with pre-symptomatic individuals, both when all samples were analysed $(4.33 \pm 0.59 \mathrm{AU} / \mathrm{ml})$ (Fig. 1b) and when only the sample closest to disease onset was analysed (5.56 $\pm 0.89 \mathrm{AU} / \mathrm{ml}$; data not shown); both comparisons were analysed at group level $(p<0.001)$. Antibody concentrations in both RA patients and pre-symptomatic individuals were also significantly increased compared with controls $(2.36 \pm 0.58 \mathrm{AU} / \mathrm{ml}, p<0.001)$ (Fig. 1b).

The frequency of anti-CPP3 antibodies was $4.5 \%$ in pre-symptomatic individuals when calculated for all 422 samples, or $6.8 \%$ when calculated for the 251 individuals who were ever positive, and $7.8 \%$ in RA patients (data not shown). Less than $2 \%$ of all individuals showed reactivity towards the arginine-containing control peptide RPP3 (data not shown).

Anti-RgpB IgG levels increased over time until symptom onset of RA, with a significant increase observed when analysing individuals with four consecutive pre-dating samples $(p<0.05$; data not shown). There was a trend towards

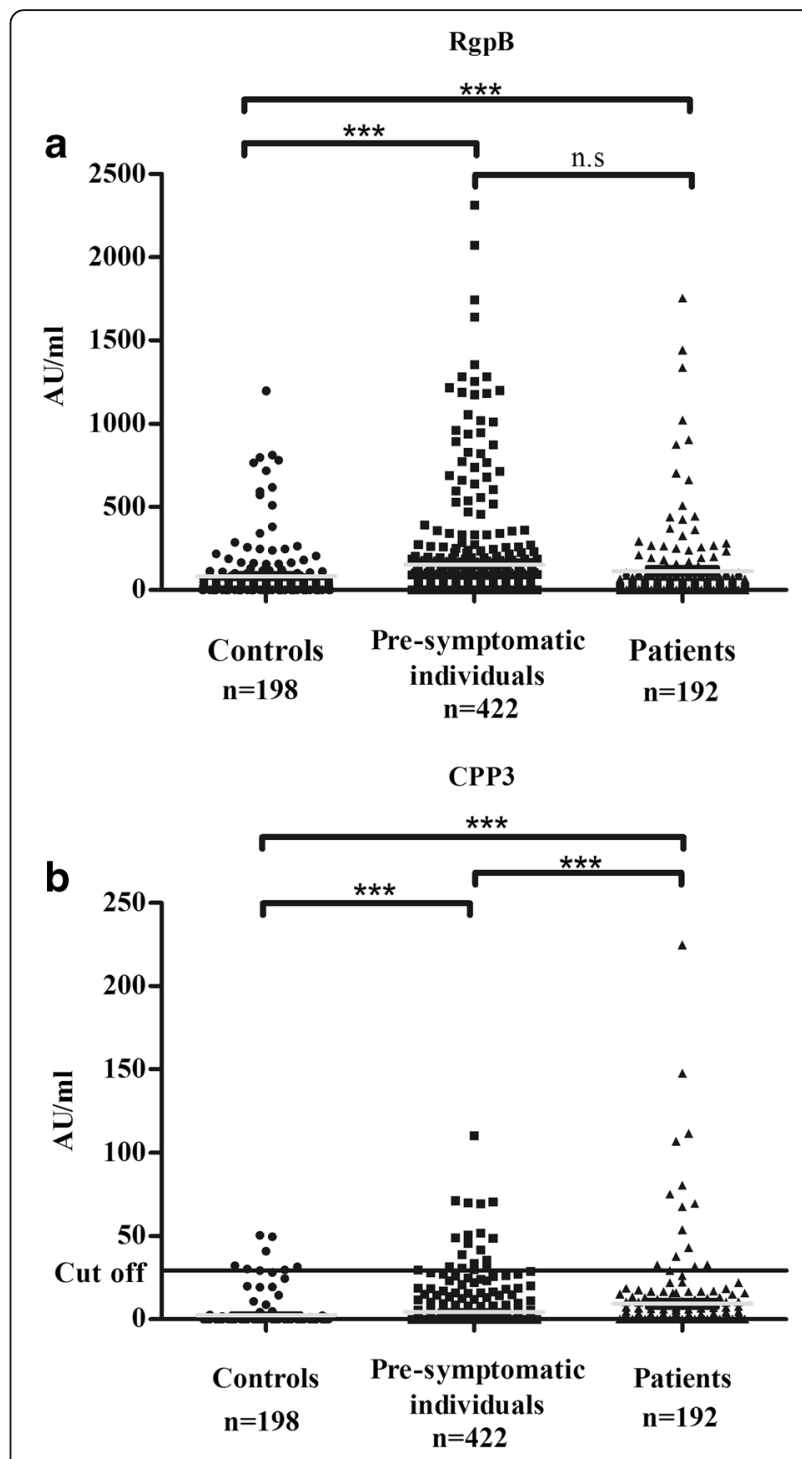

Fig. 1 Concentrations of anti-RgpB (a) and anti-CPP3 (b) antibodies in controls, pre-symptomatic individuals and patients with RA. Mean concentrations marked as a grey line. ${ }^{* *} p<0.001$. n.s not significant

lower levels following diagnosis of RA $(p<0.088)$ (Fig. 2a). Similar to the anti-RgpB IgG response, the levels of antiCPP3 IgG were found to increase constantly over the predating time (Fig. 2a). However, no relationship was found between anti-CPP3 antibody positivity and the levels of anti-RgpB IgG (data not shown). The mean concentration of anti-RgpB antibodies in pre-symptomatic individuals $(n=64)$ exceeded that of the controls already 12 years before symptom onset, whilst the corresponding time for anti-CPP3 $(n=126)$ was 8 years before symptom onset. However, anti-CPP3 antibody concentrations above the mean value of the controls were detected in 11 out of 64 (17.2\%) pre-symptomatic individuals more 

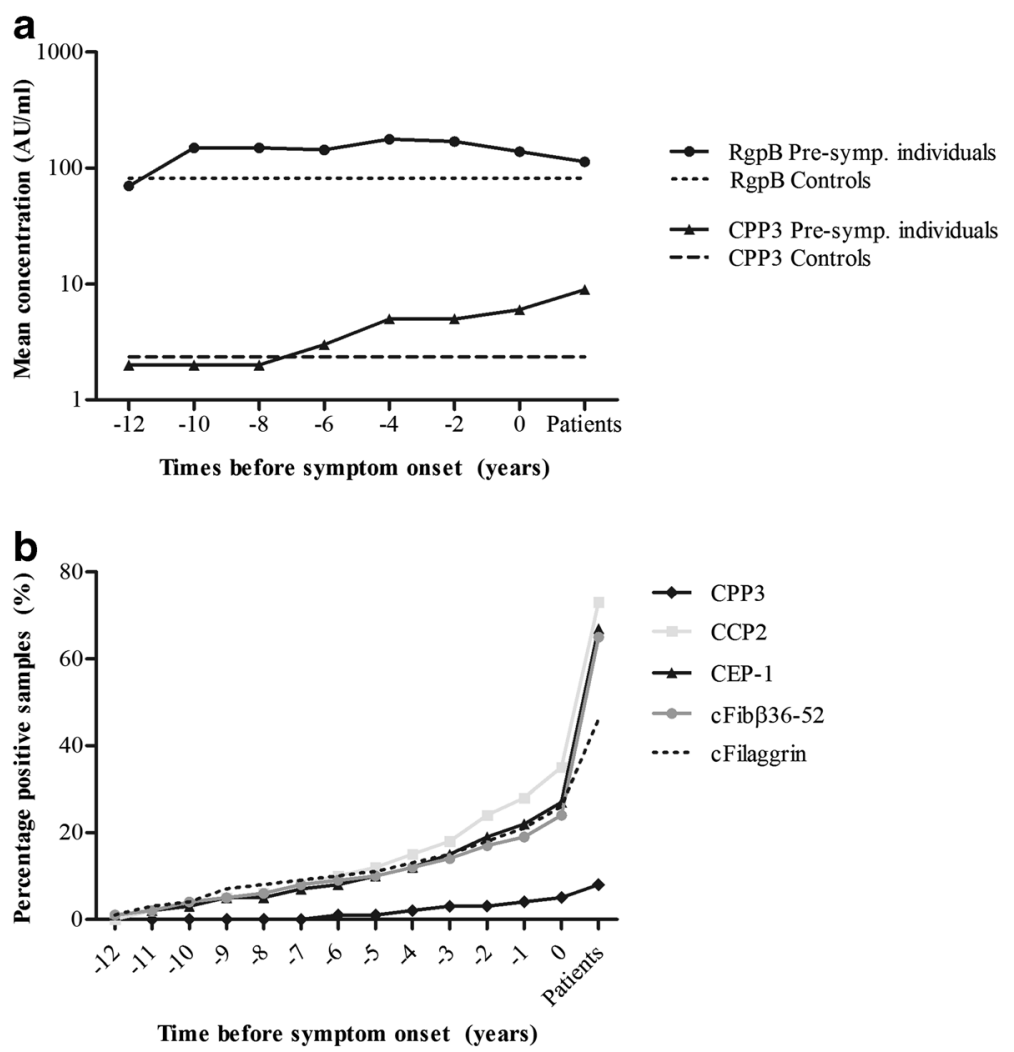

Fig. 2 Antibody responses during the pre-dating time until the time point for onset of symptoms of RA, from pre-symptomatic individuals who donated multiple blood samples pre-dating the onset of symptoms of RA $(n=422)$ and from patients with RA $(n=192)$. Logarithmic mean concentrations during 2-year periods of anti-RgpB and anti-CPP3 antibodies in pre-symptomatic individuals, patients with RA and controls $(n=198)$ (a). Accumulated percentages of antibody positivity for anti-CPP3, anti-CCP2, anti-cfibrinogen $\beta 36-52$ (cFib $\beta 36-52$ ), anti-CEP-1 (a-enolase) and anti-cfilaggrin (cFilaggrin) antibodies in pre-symptomatic individuals and in patients with RA (b). 0 time point for onset of RA symptoms

than 10 years before symptom onset. A significantly higher number of pre-symptomatic individuals (26/64, i.e. $40.6 \%$ ) had a concentration of anti-RgpB antibodies above the mean value of controls at this time point $(p<0.001)$.

\section{The anti-CPP3 and anti-RgpB antibody response in relation to the ACPA response}

The accumulated frequency of anti-CPP3 antibody positivity increased constantly over time until symptom onset (Fig. 2b). This pattern mimics that of the "classical" ACPA response (defined as antibodies against CCP2, CEP-1, cFibß36-52 and cfilaggrin) from the same time points, although at a lower frequency (Fig. 2b). The majority of anti-CPP3 IgG-positive RA patients (11 positive/15 analysed) and also pre-symptomatic individuals (11 positive/17 analysed) were confined to the antiCCP2-positive subset (Fig. 3a, b, and Additional file 1: Table S1). In pre-symptomatic individuals, anti-CPP3 positivity was associated with positivity for anti-cFib 336 52 antibodies $(\mathrm{OR}=3.22$; $95 \%$ CI 1.24-8.36, $p<0.05)$, and anti-CPP3 antibody levels correlated with the concentrations of both anti-CCP2 $\left(r_{\mathrm{s}}=0.14, p<0.01\right)$ and anti-CEP-1 antibodies $\left(r_{\mathrm{s}}=0.11, p<0.05\right)$. The median pre-dating time for anti-CPP3 antibody positivity was closer to onset ( -3.42 years) compared with antiCCP2 (-4.56 years), anti-cFibß36-52 (-5.17 years) and anti-CEP-1 ( -3.49 years) antibody positivity. There was also a significant correlation between anti-RgpB IgG levels and anti-CEP-1 antibodies $\left(r_{\mathrm{s}}=0.10, p<0.05\right)$ in pre-symptomatic individuals (data not shown). No significant relationships were found between anti-RgpB or anti-CPP3 antibodies, respectively, and RF in the presymptomatic individuals or in RA patients (data not shown).

\section{Anti-RgpB and anti-CPP3 antibody responses in relation to cigarette smoking and RA risk genes}

No associations were detected between anti-RgpB antibody levels and ever smoking in pre-symptomatic individuals, calculated in cases for whom several measurements were available or for the highest values of anti-RgpB antibodies (data not shown). In RA patients, both ever 


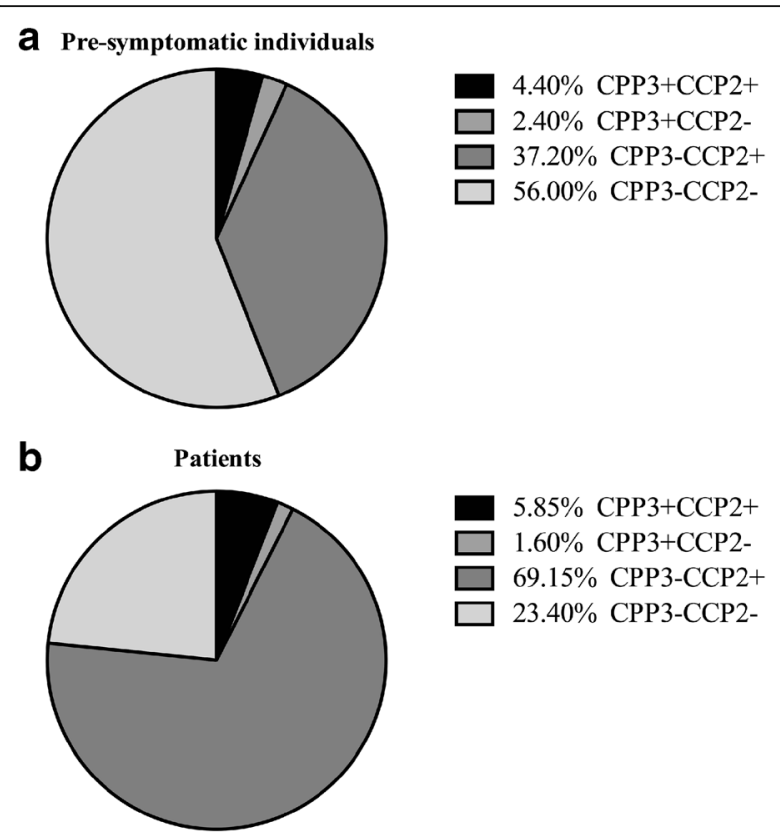

Fig. 3 Pie charts illustrating the different sub-groups of pre-symptomatic individuals (one sample per individual, $n=251$ ) (a) and RA patients ( $n=188)(\mathbf{b})$, based on the presence/absence of anti-CCP2 and anti-CPP3 antibodies. Data presented as percentage

smoking and current smoking was associated with significantly lower levels of anti-RgpB antibodies $(p<0.012$ and $p<0.019$, respectively). No associations were identified between smoking and anti-CPP3 IgG positivity in presymptomatic individuals, or in RA patients (data not shown). Moreover, no associations were observed between carriage of HLA-DRB1 SE or PTPN22 T-variant and levels of anti-RgpB or anti-CPP3 IgG positivity, in pre-symptomatic individuals. In RA, HLA-DRB1 SE was unrelated to the antibodies, while the PTPN22 Tvariant was associated with lower levels of anti-RgpB antibodies $(p<0.05$; data not shown). However, caution should be taken when interpreting these data due to the low statistical power.
Anti-RgpB and anti-CPP3 antibodies in relation to the development of RA

An association was identified between anti-RgpB antibody levels, stratified for above the 75th percentile vs below, in pre-symptomatic individuals $(\mathrm{OR}=2.31 ; 95 \%$ CI 1.41-3.78, $p<0.001$ ) (Table 2). Analyses for ever smoking or carriage of HLA-DRB1 SE or the PTPN22 Tvariant did not affect the OR (Table 2). Adjustments for age and sex in each of these analyses did not change the ORs (data not shown). Levels of anti-RgpB antibodies were not associated with having $\mathrm{RA}(\mathrm{OR}=1.20$; $95 \% \mathrm{CI}$ $0.75-1.92, p=0.44)$. Adjustments for smoking or $H L A$ DRB1 SE or the PTPN22 T-variant did not affect the association between anti-RgpB antibodies and RA (Table 2), and neither did further adjustments regarding sex and age (data not shown).

Anti-CPP3 antibodies were not significantly associated with the development of RA in pre-symptomatic individuals, irrespective of analyses including smoking or $H L A$ DRB1 SE or the PTPN22 T-variant (data not shown) or with further adjustments for sex and age (data not shown). However, anti-CPP3 IgG was associated with RA, but only when adjusting for age, sex and HLADRB1 SE (OR $=3.12$; $95 \%$ CI 1.06-9.19, $p<0.039)$ or the PTPN22 T-variant (OR = 2.96; $95 \%$ CI 1.02-8.57, $p$ $<0.045$ ) (data not shown). Adjustment for smoking, in addition to sex and age, was non-significant $(\mathrm{OR}=2.66$; $95 \%$ CI 0.97-7.26, $p=0.056)$.

Anti-CPP3 antibodies in combination with smoking or risk genes in the development of RA

When combining the major genetic risk factor for RA (HLA-DRB1 SE) with anti-CPP3 IgG positivity, an increasing risk was observed for being pre-symptomatic $(\mathrm{OR}=6.74 ; 95 \% \mathrm{CI} 1.43-31.81)$ compared with HLADRB1 SE-positive/anti-CPP3-negative individuals (OR = 3.55; 95 \% CI 2.32-5.42), although no significant interaction between the two factors was found (Table 3). Smoking in combination with anti-CPP3 antibody positivity showed no association with being pre-symptomatic $(\mathrm{OR}=2.83 ; 95 \%$ CI 0.86-9.4) (Table 3).

Table 2 Associations between anti-RgpB lgG (stratified for above the 75th percentile vs below) and the development of RA in pre-symptomatic individuals and RA patients

\begin{tabular}{|c|c|c|c|c|c|c|c|c|}
\hline & \multicolumn{4}{|c|}{ Pre-symptomatic individuals $(n=251)$} & \multicolumn{4}{|c|}{ RA patients $(n=192)$} \\
\hline & $\begin{array}{l}\text { Simple logistic } \\
\text { regression }\end{array}$ & Multiple logistic $r$ & egression & & $\begin{array}{l}\text { Simple logistic } \\
\text { regression }\end{array}$ & Multiple logistic & egression & \\
\hline Variable & OR $(95 \% \mathrm{Cl})$ & OR $(95 \% \mathrm{Cl})$ & & & OR (95 \% Cl) & OR $(95 \% \mathrm{Cl})$ & & \\
\hline Anti-RgpB & $2.31(1.41-3.78)$ & $2.24(1.35-3.71)$ & $2.55(1.67-4.43)$ & $2.17(1.28-3.68)$ & $1.20(0.75-1.92)$ & $1.18(0.73-1.92)$ & $1.30(0.77-2.17)$ & $1.22(0.74-2.00)$ \\
\hline Ever smoker & & $1.88(1.26-2.78)$ & & & & $2.14(1.41-3.26)$ & & \\
\hline HLA-DRB1 SE & & & $3.58(2.36-5.42)$ & & & & $3.14(2.05-4.83)$ & \\
\hline PTPN22 1858 T-variant & & & & $2.17(1.36-3.48)$ & & & & $2.16(1.33-3.53)$ \\
\hline
\end{tabular}


Table 3 Association of smoking or HLA-DRB1 SE and anti-CPP3 lgG in pre-symptomatic individuals compared with controls adjusted for age and sex

\begin{tabular}{lllll}
\hline & Anti-CPP3 lgG & Pre-symptomatic individuals, $n(\%)$ & Controls, $n(\%)$ & OR $(95 \% \mathrm{Cl})$ \\
\hline Smoking $^{\text {a }}$ & & & & \\
- & - & $84(33.6)$ & $90(49.7)$ & Reference \\
+ & - & $149(59.6)$ & $85(47.0)$ & $2.12(1.39-3.23)$ \\
- & + & $6(2.4)$ & $2(1.1)$ & $3.01(0.58-15.47)$ \\
+ & + & $11(4.4)$ & $4(2.2)$ & $2.83(0.86-9.40)$ \\
HLA-DRB1 SE & & & & \\
- & - & $79(31.6)$ & $107(62.6)$ & Reference \\
+ & - & $154(61.6)$ & $59(34.5)$ & $3.55(2.32-5.42)$ \\
- & + & $6(2.4)$ & $3(1.8)$ & $2.41(0.58-10.13)$ \\
+ & + & $11(4.4)$ & $2(1.2)$ & $6.74(1.43-31.81)$
\end{tabular}

$\mathrm{RERI}=2.21(-8.13,29.19), \mathrm{AP}=0.30(-3.22,0.55), \mathrm{SI}=1.52(0.22-10.90), \mathrm{Ml}=0.77(0.10-6.29), p$ value $=0.81$

aDefined as ever smoker

${ }^{\mathrm{b}}$ HLA-DRB1 shared epitope (SE) defined as 0101/0401/0404/0405/0408

$O R$ odds ratio, $\mathrm{Cl}$ confidence interval, $R E R /$ relative excess due to interaction, $A P$ attributable proportion due to interaction, $S /$ synergy index, $M I$ multiplicative interaction

In RA patients, smoking combined with anti-CPP3 IgG increased the OR significantly from 1.73 (95\% CI 1.10-2.72) in anti-CPP3 IgG-negative ever smokers to 3.61 (95 \% CI 1.05-12.44) in anti-CPP3 IgG-positive ever smokers (Table 4). HLA-DRB1 SE also yielded significantly higher OR in combination with anti-CPP3 IgG positivity than in combination with anti-CPP3 negativity $(\mathrm{OR}=8.80 ; 95 \% \mathrm{CI} 1.80-43.03$ vs $\mathrm{OR}=3.33 ; 95 \% \mathrm{CI}$ 2.11-5.23) (Table 4). However, no significant interactions were observed between smoking and anti-CPP3 IgG, or between SE and anti-CPP3 IgG. Carriage of the PTPN22 T-variant in combination with anti-CPP3 antibody positivity revealed no significant association with the development of RA in patients or pre-symptomatic individuals (data not shown). Current smoking yielded similar results to ever smoking, although slightly weaker associations, which could be due to fewer current smokers compared with ever smokers, which also include former smokers.

\section{Discussion}

In the present study we investigated the role of the oral pathogen $P$. gingivalis in the development of RA, by focusing on the anti- $P$. gingivalis antibody response in RA patients prior to onset of symptoms. Our recent data show elevated antibody levels against the potent $P$. gingivalis virulence factor arginine gingipainB in patients with RA, especially in ACPA-positive RA [21]. We have

Table 4 Association of smoking or HLA-DRB1 SE and anti-CPP3 IgG in patients with RA compared with controls adjusted for age and sex

\begin{tabular}{lllll}
\hline & Anti-CPP3 IgG & RA patients, $n(\%)$ & Controls, $n(\%)$ & OR (95 \% Cl) \\
\hline Smoking $^{a}$ & & & & \\
- & - & $58(30.2)$ & $90(49.7)$ & Ref. \\
+ & - & $119(62.0)$ & $85(47.0)$ & $1.73(1.10-2.72)$ \\
- & + & $5(2.6)$ & $2(1.1)$ & $4.04(0.73-22.32)$ \\
+ & + & $10(5.2)$ & $4(2.2)$ & $3.61(1.05-12.44)$
\end{tabular}

$\mathrm{RERI}=-1.17(-18.21,8.19), \mathrm{AP}=-0.30(-7.38,0.46), \mathrm{SI}=0.71(0.08-6.55), \mathrm{MI}=0.46(0.059-3.59), p$ value $=0.46$

HLA-DRB1 SE ${ }^{\mathrm{b}}$

$\begin{array}{lllll}- & - & 64(33.3) & 107(62.6)) & \text { Ref. } \\ + & - & 113(58.9) & 59(34.5) & 3.33(2.11-5.23) \\ - & + & 6(3.1) & 3(1.8) & 3.55(0.84-15.03) \\ + & + & 9(4.7) & 2(1.2) & 8.80(1.80-43.03)\end{array}$

$\mathrm{RERI}=1.98(-10.10,30.25), \mathrm{AP}=0.26(-3.96,0.57), \mathrm{SI}=1.44(0.18-11.19), \mathrm{MI}=0.70(0.08-5.81), p$ value $=0.74$ 
also shown that these antibodies are clearly elevated in patients with PD, compared with periodontally healthy individuals, demonstrating that anti-RgpB IgG probably represents a good surrogate marker for $P$. gingivalis infection, which has been associated with PD [22, 23]. With the present study, we report increased concentrations of these antibodies in a subset of individuals years before onset of symptoms of RA. Consistent with Quirke et al.'s data in RA [25], concentrations of anti-CPP3 antibodies directed against, a synthetic cyclic citrullinated peptide derived from another $P$. gingivalis-specific virulence factor, P.PAD, were also increased in a subset of both pre-symptomatic individuals and RA patients, compared with controls.

In line with our previous findings in RA patients, the association between the development of RA in presymptomatic individuals and the anti-RgpB antibody response was not dependent on smoking habits or presence of $H L A-D R B 1$ SE or the PTPN22 T-variant [21]. Our data on lower anti-RgpB IgG levels in RA patients who were ever smokers or current smokers compared with non-smokers were also in line with a number of previous reports showing lower anti- $P$. gingivalis antibody levels in smokers compared with non-smokers $[21,39,40]$. One explanation for the observed trend of lower anti-RgpB antibody levels in RA patients compared with pre-symptomatic individuals (Figs. 1a and 2a) could therefore potentially be the higher frequency of smokers among RA patients (67.2 \%), compared with presymptomatic individuals $(64 \%)$. Furthermore, as we recently showed for the anti-RgpB IgG response in RA, the HLA-DRB1 SE in combination with anti-CPP3 IgG reveals a stronger association with $\mathrm{RA}$ and with being pre-symptomatic than $H L A-D R B 1 \mathrm{SE}$ alone. The same effect occurred when combining smoking with antiCPP3 IgG positivity, although only in RA patients, not in pre-symptomatic individuals.

In this study, stratification of data into ACPA-positive and ACPA-negative sub-groups was not possible due to the limited number of individuals. However, a weak correlation between the concentration of anti-RgpB antibodies and that of the "classical" ACPA, measured as anti-CEP-1 antibodies, could be observed. Notably, ACPA (anti-CEP-1, anti-cFibß36-52 and anti-cfilaggrin antibodies) were analysed by the ISAC multiplex assay, which is only a semiquantitative method [37], and thus are not completely comparable with results from the ELISA used for measuring anti-RgpB IgG.

No relationship was detected between anti-RgpB and anti-CPP3 antibodies. This was unexpected considering the origin of both $\mathrm{RgpB}$ and CPP3 as P. gingivalis-specific antigens, and our interpretation of these two antibodies as surrogate markers for a $P$. gingivalis infection. Although the concentrations of anti-CPP3 antibodies, like anti-RgpB antibodies, were significantly increased in both RA patients and pre-symptomatic individuals compared with controls, the frequency of anti-CPP3 antibodies was only significantly increased in RA patients. Moreover, the anti-RgpB antibody response was elevated (compared with controls) several years earlier (12 years) than the anti-CPP3 antibody response (8 years). The antiCPP3 antibody response was similar to the "classical" ACPA response; that is, there was no reactivity to the arginine-containing control peptide RPP3, the majority of anti-CPP3 antibody-positive cases were also anti-CCP2 antibody positive and the anti-CPP3 antibody response (both concentrations and the accumulated frequency of positive samples) increased gradually during the pre-dating time until symptom onset [37]. Taken together, these data may suggest that the anti-CPP3 antibody response, rather than being $P$. gingivalis specific, simply belongs to the generic ACPA response, or rather represents cross-reactivity with another citrullinated antigen. The low frequency of these antibodies ( $8 \%$ in RA) could point to this. Moreover, in-vivo auto-citrullination by P.PAD has been debated [39], and the CPP3/RPP3 peptide-with its internal rather than C-terminal citrulline residue-may not represent an in-vivo-generated antigen. Still, the CPP3/RPP3 peptide sequence is bacterial derived and does not correspond to any human protein sequence.

Results recently published by Fisher et al. [41] conflict with ours in that they were unable to identify associations between anti-RgpB or anti-CPP3 IgG and pre RA. The two studies differ in several aspects that may explain the discrepant results: their study was based on a smaller study population $(n=103)$ than ours $(n=251$, with 422 samples), with participants from a number of different southern European countries whilst our study subjects were recruited from a geographically defined area in northern Sweden; and not all of the pre-symptomatic individuals in Fisher et al.'s study were confirmed to develop RA, as they were in our study [41]. Additionally, the periodontal microbiota has been shown to vary between countries [42] and bacterial strain diversity has been described previously for $P$. gingivalis [43]. Altogether, these differences could contribute to the variability in the results of these studies.

Supporting our results, Mikuls et al. [44] reported increased concentrations of anti- $P$. gingivalis antibodies in high-risk individuals compared with controls. Also in accordance with our data is a study by de Smit et al. [45], in which elevated anti- $P$. gingivalis antibody levels were observed in arthralgia patients with RF or ACPA positivity compared with controls. However, anti-P. gingivalis antibody levels in de Smit et al.'s study were not higher in arthralgia individuals who developed RA compared with those who did not. This was not possible to evaluate in our study [45]. 
We believe this to be the largest population-based study analysing the anti- $P$. gingivalis antibody response in individuals before onset of symptoms of RA performed to date. Our study has limitations: the samples analysed were from different population surveys, and were not collected on a regular basis; no information regarding periodontal status or treatment was available, hence there was an inability to set a cut-off value for the anti-RgpB antibodies response; and it was not possible to investigate the relationship between $\mathrm{PD}$ and the development of RA. Also, data on the presence of $P$. gingivalis DNA were not available, and analysis of the anti-RgpB IgG and the anti-CPP3 IgG responses in relation to the presence of the bacteria was consequently not possible. However, as in our previous study [21], the anti-RgpB IgG response was interpreted as a surrogate marker for a $P$. gingivalis infection, past or present, whilst our data suggest that the anti-CPP3 antibody response, which follows the "classical" ACPA response, should be considered ACPA specific rather than a $P$. gingivalis-specific antibody.

\section{Conclusions}

Our data demonstrate that antibodies against $P$. gingivalis are significantly increased in patients with RA compared with controls, and that these antibodies are detectable years before the onset of symptoms, supporting an aetiological role for $P$. gingivalis in the development of RA. Studies on larger cohorts with samples collected on a regular basis are needed for a deeper understanding of the relationship between $P$. gingivalis, anti- $P$. gingivalis antibodies, ACPA and the development of RA.

\section{Additional file}

Additional file 1: Table S1. Presenting the frequency of ever positivity for anti-CPP3 antibodies in relation to positive/negative anti-CCP2 antibodies or ACPA. (DOCX $15 \mathrm{~kb}$ )

\section{Acknowledgements}

The authors would like to thank the study participants; the staff involved in the collection of blood samples; Professor Göran Hallmans at the Department of Public Health and Clinical Medicine, Nutritional Research, University Hospital, Umeå for providing samples from the Medical Biobank; and Professor Lars Klareskog, Karolinska Institutet, Stockholm, Professor Johan Rönnelid, Uppsala University, Uppsala and Mrs Monika Hansson, PhD, Karolinska Institutet, Stockholm as well as Thermo Fisher Scientific Sweden for collaboration with the antibody multiplex analysis.

\section{Funding}

This study was supported by grants from the Swedish Rheumatism Association and the Swedish Research Council (K2013-52X-20307-07-3). SR-D was also supported by grants from King Gustaf V's 80-Year Fund, the Västerbotten county council (ALF) and the Swedish Foundation for Strategic Research, Sweden. KL was also supported by grants from the EU-funded FP7 project TRIGGER (FP7-Health-2013-306029) and the ITN project Rapid (290246).

\section{Authors' contributions}

$\mathrm{U}$ and NS made substantial contributions to the conception and design, and acquisition, analysis and interpretation of data, and were involved in drafting the manuscript or revising it critically for important intellectual content. NK was involved in revising the manuscript critically for important intellectual content, and was involved in analysing and calculating the data. BP was involved in revising the manuscript critically for important intellectual content and has agreed to be accountable for all aspects of the work in ensuring that questions related to the accuracy or integrity of any part of the work are appropriately investigated and resolved. LI was involved in analysing and calculating the data and participated in the discussions concerning the manuscript. BL as involved in analysing and calculating the data and participated in the discussions concerning the manuscript. JP was involved in revising the manuscript critically for important intellectual content and has agreed to be accountable for all aspects of the work in ensuring that questions related to the accuracy or integrity of any part of the work are appropriately investigated and resolved. SR-D and KL made substantial contributions to the conception and design, and acquisition, analysis and interpretation of data, and have agreed to be accountable for all aspects of the work in ensuring that questions related to the accuracy or integrity of any part of the work are appropriately investigated and resolved. All authors read and approved the final manuscript.

\section{Authors' information}

$L J$ and NS share first authorship. SRD and KL share senior authorship.

\section{Competing interests}

The authors declare that they have no competing interests.

\section{Ethical approval and consent to participate}

The participants gave their written informed consent and the Regional Ethics Committee at Umeå University approved the study.

\section{Author details}

${ }^{1}$ Public Health and Clinical Medicine/Rheumatology, Umeå University, Umeå, Sweden. ${ }^{2}$ Rheumatology Unit, Department of Medicine Solna, Karolinska Institutet, Stockholm, Sweden. ${ }^{3}$ Department of Oral Immunology and Infectious Diseases, University of Louisville, School of Dentistry, Louisville, KY, USA. ${ }^{4}$ Department of Microbiology, and Malopolska Centre of Biotechnology, Faculty of Biochemistry, Biophysics and Biotechnology, Jagiellonian

University, Krakow, Poland.

Received: 1 July 2016 Accepted: 22 August 2016

Published online: 07 September 2016

\section{References}

1. Neovius M, Simard JF, Askling J, ARTIS study group. Nationwide prevalence of rheumatoid arthritis and penetration of disease-modifying drugs in Sweden. Ann Rheum Dis. 2011;70:624-9.

2. Harris Jr ED. Rheumatoid arthritis. Pathophysiology and implications for therapy. N Engl J Med. 1990;322:1277-89.

3. Firestein GS. Evolving concepts of rheumatoid arthritis. Nature. 2003;423: 356-61.

4. van Venrooij WJ, Hazes JM, Visser H. Anticitrullinated protein/peptide antibody and its role in the diagnosis and prognosis of early rheumatoid arthritis. Neth J Med. 2002;60:383-8.

5. Klareskog L, Catrina Al, Paget S. Rheumatoid arthritis. Lancet. 2009;373:659-72.

6. McInnes IB, Schett $\mathrm{G}$. The pathogenesis of rheumatoid arthritis. N Engl J Med. 2011:365:2205-19.

7. Williams RC. Periodontal disease. N Engl J Med. 1990;322:373-82.

8. de Pablo P, Dietrich T, McAlindon TE. Association of periodontal disease and tooth loss with rheumatoid arthritis in the US population. J Rheumatol. 2008:35:70-6.

9. Demmer RT, Molitor JA, Jacobs Jr DR, Michalowicz BS. Periodontal disease, tooth loss and incident rheumatoid arthritis: results from the First National Health and Nutrition Examination Survey and its epidemiological follow-up study. J Clin Periodontol. 2011;38:998-1006.

10. Mikuls TR, Payne JB, Yu F, et al. Periodontitis and Porphyromonas gingivalis in patients with rheumatoid arthritis. Arthritis Rheumatol. 2014;66:1090-100.

11. Scher JU, Ubeda C, Equinda M, et al. Periodontal disease and the oral microbiota in new-onset rheumatoid arthritis. Arthritis Rheum. 2012;64:3083-94. 
12. Bostrom EA, Kindstedt E, Sulniute R, et al. Increased eotaxin and MCP-1 levels in serum from individuals with periodontitis and in human gingival fibroblasts exposed to pro-inflammatory cytokines. PLoS One. 2015;10, e0134608.

13. Ebersole JL, Machen RL, Steffen MJ, Willmann DE. Systemic acute-phase reactants, C-reactive protein and haptoglobin, in adult periodontitis. Clin Exp Immunol. 1997;107:347-52.

14. Katz J, Goultschin J, Benoliel R, Brautbar C. Human leukocyte antigen (HLA) DR4. Positive association with rapidly progressing periodontitis. J Periodontol. 1987;58:607-10.

15. Heliövaara M, Aho K, Aromaa A, Knekt $P$, Reunanen A. Smoking and risk of rheumatoid arthritis. J Rheumatol. 1993;20:1830-5.

16. Tonetti MS. Cigarette smoking and periodontal diseases: etiology and management of disease. Ann Periodontol. 1998;3:88-101.

17. Harvey GP, Fitzsimmons TR, Dhamarpatni AA, et al. Expression of peptidylarginine deiminase-2 and -4 , citrullinated proteins and anticitrullinated protein antibodies in human gingiva. J Periodontal Res. 2013; 48:252-61.

18. Schellekens GA, Visser $H$, de Jong BA, et al. The diagnostic properties of rheumatoid arthritis antibodies recognizing a cyclic citrullinated peptide. Arthritis Rheum. 2000:43:155-63.

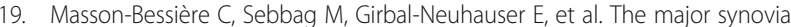
targets of the rheumatoid arthritis-specific antifilaggrin autoantibodies are deiminated forms of the alpha- and beta-chains of fibrin. J Immunol. 2001; 166:4177-84.

20. Eriksson K, Nise L, Kats A, et al. Prevalence of Periodontitis in Patients with Established Rheumatoid Arthritis: a Swedish population based case-control study. PLoS One. 2016;11, e0155956.

21. Kharlamova $N$, Jiang $X$, Sherina $N$, et al. Antibodies to Porphyromonas gingivalis indicate interaction between oral infection, smoking and risk genes in rheumatoid arthritis etiology. Arthritis Rheumatol. 2016;68:604-13.

22. Socransky SS, Haffajee AD, Cugini MA, Smith C, Kent Jr RL. Microbial complexes in subgingival plaque. J Clin Periodontol. 1998;25:134-44.

23. Hajishengallis G. Periodontitis: from microbial immune subversion to systemic inflammation. Nat Rev Immunol. 2015:15:30-44.

24. McGraw WT, Potempa J, Farley D, Travis J. Purification, characterization, and sequence analysis of a potential virulence factor from Porphyromonas gingivalis, peptidylarginine deiminase. Infect Immun. 1999;67:3248-56.

25. Quirke AM, Lugli EB, Wegner $N$, et al. Heightened immune response to autocitrullinated Porphyromonas gingivalis peptidylarginine deiminase: a potential mechanism for breaching immunologic tolerance in rheumatoid arthritis. Ann Rheum Dis. 2014;73:263-9.

26. Wegner N, Wait R, Sroka A, et al. Peptidylarginine deiminase from Porphyromonas gingivalis citrullinates human fibrinogen and alpha-enolase: implications for autoimmunity in rheumatoid arthritis. Arthritis Rheum. 2010; 62:2662-72

27. Rosenstein ED, Greenwald RA, Kushner LJ, Weissmann G. Hypothesis: the humoral immune response to oral bacteria provides a stimulus for the development of rheumatoid arthritis. Inflammation. 2004;28:311-8.

28. Lundberg K, Kinloch A, Fisher BA, et al. Antibodies to citrullinated alphaenolase peptide 1 are specific for rheumatoid arthritis and cross-react with bacterial enolase. Arthritis Rheum. 2008;58:3009-19.

29. Lundberg K, Wegner N, Yucel-Lindberg T, Venables PJ. Periodontitis in RA-the citrullinated enolase connection. Nat Rev Rheumatol. 2010:6:727-30.

30. Rantapaa-Dahlqvist S, de Jong BA, Berglin E, et al. Antibodies against cyclic citrullinated peptide and IgA rheumatoid factor predict the development of rheumatoid arthritis. Arthritis Rheum. 2003;48:2741-9.

31. Nielen MM, van Schaardenburg D, Reesink HW, et al. Specific autoantibodies precede the symptoms of rheumatoid arthritis: a study of serial measurements in blood donors. Arthritis Rheum. 2004;50:380-6.

32. Willis VC, Demoruelle MK, Derber LA, et al. Sputum autoantibodies in patients with established rheumatoid arthritis and subjects at risk of future clinically apparent disease. Arthritis Rheum. 2013;65:2545-54.

33. Arnett FC, Edworthy SM, Bloch DA, et al. The American Rheumatism Association 1987 revised criteria for the classification of rheumatoid arthritis. Arthritis Rheum. 1988;31:315-24.

34. Berglin E, Padyukov L, Sundin U, et al. A combination of autoantibodies to cyclic citrullinated peptide (CCP) and HLA-DRB1 locus antigens is strongly associated with future onset of rheumatoid arthritis. Arthritis Res Ther. 2004; 6:R303-8.

35. Johansson M, Arlestig L, Hallmans G, Rantapää-Dahlqvist S. PTPN22 polymorphism and anti-cyclic citrullinated peptide antibodies in combination strongly predicts future onset of rheumatoid arthritis and has a specificity of $100 \%$ for the disease. Arthritis Res Ther. 2006:8:R19.

36. Potempa J, Nguyen KA. Purification and characterization of gingipains. Curr Protoc Protein Sci. 2007; Chapter 21:Unit 21.20.

37. Brink M, Hansson M, Mathsson L, et al. Multiplex analyses of antibodies against citrullinated peptides in individuals prior to development of rheumatoid arthritis. Arthritis Rheum. 2013:65:899-910.

38. Zou GY. On the estimation of additive interaction by use of the four-by-two table and beyond. Am J Epidemiol. 2008:168:212-24.

39. Vlachojannis C, Dye BA, Herrera-Abreu M, et al. Determinants of serum lgG responses to periodontal bacteria in a nationally representative sample of US adults. J Clin Periodontol. 2010;37:685-96.

40. Seror R, Le Gall-David S, Bonnaure-Mallet M, et al. Association of anti Porphyromonas gingivalis antibody titers with nonsmoking status in early rheumatoid arthritis: results from the prospective French cohort of patients with early rheumatoid arthritis. Arthritis Rheumatol. 2015;67:1729-37.

41. Fisher BA, Cartwright AJ, Quirke AM, et al. Smoking, Porphyromonas gingivalis and the immune response to citrullinated autoantigens before the clinical onset of rheumatoid arthritis in a Southern European nested case-control study. BMC Musculoskelet Disord. 2015;16:331.

42. Haffajee AD, Bogren A, Hasturk $H$, et al. Subgingival microbiota of chronic periodontitis subjects from different geographic locations. J Clin Periodontol. 2004;31:996-1002.

43. Sundqvist G, Figdor D, Hänström L, Sörlin S, Sandström G. Phagocytosis and virulence of different strains of Porphyromonas gingivalis. Scand J Dent Res. 1991;99:117-29.

44. Mikuls TR, Thiele GM, Deane KD, et al. Porphyromonas gingivalis and disease related autoantibodies in individuals at increased risk of rheumatoid arthritis. Arthritis Rheum. 2012;64:3522-30.

45. de Smit M, van de Stadt LA, Janssen KMJ, et al. Antibodies against Porphyromonas gingivalis in seropositive arthralgia patients do not predict development of rheumatoid arthritis. Ann Rheum Dis. 2014;73:1277-9.

\section{Submit your next manuscript to BioMed Central and we will help you at every step:}

- We accept pre-submission inquiries

- Our selector tool helps you to find the most relevant journal

- We provide round the clock customer support

- Convenient online submission

- Thorough peer review

- Inclusion in PubMed and all major indexing services

- Maximum visibility for your research

Submit your manuscript at www.biomedcentral.com/submit
Biomed Central 